\title{
The 6-Minute Walk Test in Chronic Respiratory Failure: Does Observed or Predicted Walk Distance Better Reflect Patient Functional Status?
}

\author{
Gökay Güngör MD, Zuhal Karakurt MD, Nalan Adıgüzel MD, Rüya Evin Aydın PT, \\ Merih Kalamanoğlu Balcı MD, Cüneyt Saltürk MD, Raziye Sancar RN, \\ Suat Solmaz RN, and Özlem Yazıcıŏlu Moçin MD
}

\begin{abstract}
BACKGROUND: Acquiring 6-min walk test (6MWT) data from patients undergoing noninvasive mechanical ventilation due to chronic hypercapnic respiratory failure is limited. We aimed to assess whether the actual 6-min walk distance (6MWD) or the percent predicted 6MWD is a better reflection of the respiratory function of patients using home noninvasive ventilation (NIV) due to chronic hypercapnic respiratory failure. METHODS: This was a cross-sectional observational study. The 6MWT was performed in subjects using home NIV. Diagnoses were grouped as COPD, obesity hypoventilation syndrome (OHS), kyphoscoliosis, and parenchymal lung disease. Sex, age, and body mass index (BMI) were used to calculate ideal 6MWD. Male: 1,140 m - $(5.61 \times \mathrm{BMI})-$ $(6.94 \times$ age $)$, and subtract $153 \mathrm{~m}$ for the lower limit of normal. Female: $1,017 \mathrm{~m}-(6.24 \times \mathrm{BMI})-$ $(5.83 \times$ age $)$, and subtract $139 \mathrm{~m}$ for the lower limit of normal. The 6MWD and percent-ofpredicted 6MWD were compared relative to arterial blood gas, spirometry values, and diagnosis. RESULTS: The 6MWT was performed in 144 subjects, median (IQR) age 62 y $(55-71 \mathrm{y})$. The male/female ratio, median (IQR) 6MWD, and percent-of-predicted 6MWD values were: COPD $32 / 6,316 \mathrm{~m}(226-390 \mathrm{~m})$, and 59.4\% (42.5-68.9\%); OHS 24/28, $303 \mathrm{~m}(240-362 \mathrm{~m})$, and 73.0\% (63.0-82.0\%); kyphoscoliosis 16/7, $420 \mathrm{~m}(318-462 \mathrm{~m})$, and 70.5\% (56.0-75.2\%); and parenchymal lung disease 19/12, $333 \mathrm{~m}(273-372 \mathrm{~m})$, and $67.1 \%(46.7-74.7 \%)$. The correlation of percentof-predicted 6MWD with spirometry and arterial blood gas values were better than with the actual 6MWD. CONCLUSIONS: The percent-of-predicted 6MWD was better correlated with respiratory function than actual 6MWD for subjects using home NIV due to chronic hypercapnic respiratory failure with COPD, OHS, kyphoscoliosis, and parenchymal lung disease. Key words: 6-min walk test; chronic hypercapnic respiratory failure; noninvasive ventilation; walking distance. [Respir Care 2013; 58(5):850-857. (C) 2013 Daedalus Enterprises]
\end{abstract}

\section{Introduction}

The 6-min walk test (6MWT) is used for its simplicity and reproducibility, and is well tolerated when evaluating the functional status of patients. ${ }^{1}$ The 6 -min walk distance (6MWD) represents a satisfactory reflection of the physical capacity of patients. ${ }^{2}$ The actual 6MWD is used to evaluate the functional status of patients to compare the effects of therapeutic interventions and to predict mortality

\footnotetext{
Correspondence: Gökay Güngör MD, Respiratory Intensive Care Unit, Sureyyapasa Chest Diseases and Thoracic Surgery Training and Research Hospital, Istanbul 34846 Turkey. E-mail:drgokaygungor@ hotmail.com.
}

DOI: $10.4187 /$ respcare.02009

\footnotetext{
The authors are affiliated with the Respiratory Intensive Care Unit, Sureyyapasa Chest Diseases and Thoracic Surgery Training and Research Hospital, Istanbul, Turkey.

The authors presented a version of this paper at the 15th National Congress The authors have disclosed no conflicts of interest.
} 
associated with various cardiopulmonary diseases. ${ }^{3-6}$ Actual 6MWD was also studied in chronic hypercapnic respiratory failure patients who were using home noninvasive ventilation (NIV). ${ }^{7}$ Budweiser et al found a similar hazard ratio for mortality for the actual 6MWD and percent-of-predicted 6MWD in 424 patients with chronic hypercapnic respiratory failure using home NIV. ${ }^{7}$ However, they did not study the relationship between pulmonary function and actual 6MWD versus percent-of-predicted 6MWD.

Various studies have been performed since 1998 to determine a standard measure of the 6MWT, taking into account age, sex, and body mass index (BMI) in healthy adult. ${ }^{8-11}$ The reference equations for 6MWT and lung function were used in stable patients in these studies. We have previously shown that by using reference equations (age, sex, and BMI), pulmonary functions in kyphoscoliosis patients with chronic hypercapnic respiratory failure and on home NIV are better correlated with percent-ofpredicted 6MWD than with actual 6MWD. ${ }^{12}$

In current clinical practice the distance walked is the only crucial factor for physicians when making clinical judgments on patients with cardiopulmonary diseases. The actual 6MWD uses only the number of meters walked, but the walking distance is dependent on various factors such as age, sex, and BMI. In normal subjects there are a sufficient number of studies dealing with standardization of the 6MWT. The importance and relevance of the percentof-predicted 6MWD are unknown in cardiopulmonary diseases.

In the present study we used reference equations for 6MWT in a large series of chronic hypercapnic respiratory failure patients with home NIV, and assessed their value in reflecting pulmonary function. We endeavored to answer whether the observed walking distance or the percentage predicted value is a better reflection of respiratory function in patients using home NIV due to chronic hypercapnic respiratory failure.

\section{Methods}

The study was approved by the local ethical committee of our hospital; it was designed as a cross-sectional study in a large pulmonary disease-based, tertiary, training and research hospital in Istanbul, Turkey.

\section{Inclusion Criteria}

Patients treated with home NIV due to chronic hypercapnic respiratory failure, and who were regularly followed up at the respiratory intensive care out-patient clinic, were recruited consecutively into the study from June 2011 to December 2011. Additionally, a duration of nocturnal home NIV of $\geq 3$ months was required. Subjects were

\section{QUICK LOOK}

\section{Current knowledge}

The 6-min walk distance (6MWD) reflects the patient's physical capacity, and is valued for its simplicity, reproducibility, and tolerability. 6MWD is used to evaluate functional status and the effects of therapies, and to predict the risk of death.

\section{What this paper contributes to our knowledge}

Using reference equations, the 6MWD and percent of predicted 6MWD are important for assessing cardiopulmonary function. Sex is an important bias factor for 6MWD. Percent of predicted 6MWD was better related to pulmonary function than was 6MWD.

grouped according to a diagnosis of being in stable clinical condition with COPD, obesity hypoventilation syndrome (OHS), kyphoscoliosis, or parenchymal lung disease. The diagnosis of COPD was based on clinical history, symptoms, and airway obstruction (ie, $\mathrm{FEV}_{1} / \mathrm{FVC}<70 \%$ of predicted after bronchodilator inhalation). ${ }^{13} \mathrm{OHS}$ was defined by a BMI $>30 \mathrm{~kg} / \mathrm{m}^{2}$, daytime $\mathrm{P}_{\mathrm{aCO}_{2}} \geq 45 \mathrm{~mm} \mathrm{Hg}$ prior to NIV therapy, and clinical symptoms of chronic hypercapnic respiratory failure in the absence of other known causes of hypoventilation. ${ }^{14}$ Parenchymal lung disease was defined as lung parenchyma defective due to tuberculosis sequelae or bronchiectasis, which caused hypoventilation and resulted in hypoxemia and hypercapnia.

\section{Exclusion Criteria}

Patients who were disabled or unwilling to walk were excluded. Patients with clinical signs of airway infection, current exacerbation, or unstable cardiac arrhythmia were also excluded.

\section{Interventions}

The 6MWD was determined based on the American Thoracic Society ${ }^{1}$ guidelines, while the subjects had their usual oxygen flow. The test was performed in a 30-m long corridor by a physiotherapist with specific experience. The course was demarcated by 2 traffic cones, and the corridor was marked every $3 \mathrm{~m}$, according to the American Thoracic Society standards. Instructions and verbal encouragement given to the subjects were standardized. Encouragement was given every minute during the test until subject exhaustion. The end of the test was determined either by the subject, for any reason, or by the physical therapist conducting the test. Chest pain, intolerable dyspnea, diz- 
ziness leg cramps, diaphoresis, and pallor were additional criteria for immediately stopping the test. The Borg dyspnea scale ${ }^{15}$ and fatigue score ${ }^{16}$ were scored as described. Ideal 6MWT values were calculated according to formula, by using reference equations provided by Enright and Sherrill (see below). ${ }^{8}$ The test results were recorded as absolute, percent-of-predicted, and lower limit of normal values, according to previous methods 8 :

$$
\begin{aligned}
& \text { Male ideal } 6 \mathrm{MWD}= \\
& \qquad 1,140-(5.61 \times \mathrm{BMI})-(6.94 \times \text { age }) \\
& \text { Female ideal } 6 \mathrm{MWD}= \\
& \qquad 1,017-(6.24 \times \mathrm{BMI})-(5.83 \times \text { age }) \\
& \text { Percent-of-predicted } 6 \mathrm{MWD}=\% \text { of ideal } 6 \mathrm{MWD} \\
& \text { Male lower limit of normal }=\text { ideal } 6 \mathrm{MWD}-153 \mathrm{~m} \\
& \text { Female lower limit of normal }=\text { ideal } 6 \mathrm{MWD}-139 \mathrm{~m}
\end{aligned}
$$

BMI was calculated and comorbidities were documented. For kyphoscoliosis subjects, height was calculated from arm span (largest distance across the middle fingers when the arms are stretched horizontally sideways). In males, arm span/1.03, and in women, arm span/1.01 was used for standing height in the regression equation. ${ }^{17}$

\section{Protocol}

On the day of the out-patient clinic visit, arterial blood gases (ABGs) (Rapidlab, Bayer, Leverkusen, Germany) were analyzed at rest from the radial artery. Samples were taken in the daytime during spontaneous breathing of room air, if possible, or otherwise during the subject's usual oxygen flow. Oxygenation was defined $\mathrm{P}_{\mathrm{aO}_{2}} / \mathrm{F}_{\mathrm{IO}_{2}}$. Spirometry (Zan GPI 3.00, nSpire Health, Longmont, Colorado) was performed according to American Thoracic Society guidelines. ${ }^{18}$

\section{Statistical Analysis}

For data description, continuous variables are shown as median and (IQR) or number and percentage, when appropriate. Spearman rank correlation test was used to correlate sex and disease group according to respiratory function for actual 6MWD, and sex-specific regression equations for 6MWD. Residuals were defined for actual 6MWD and percent-of-predicted 6MWD as actual 6MWD minus ideal 6MWD, and observed percent-of-predicted 6MWD minus $100 \%$, respectively. Scatter plot graphs of the difference between the predicted and the actual 6MWD against the ideal 6MWD were drawn to investigate the residual distribution. This graph was necessary to assess whether the difference was uniformly distributed or varying over the range of prediction. This was based on the concept of assessing agreement between 2 methods of clin- ical measurement, which was designed by Bland-Altman. ${ }^{19}$ Residuals were standardized by subtracting differences from the mean and dividing to the standard deviation. Standardized residuals were compared between sexes to assess the potential bias in the agreement between predicted and observed 6MWD (ie, if the difference between the predicted and observed values changed with this factor). Data were analyzed using a statistical software package (SPSS 15.0, SPSS, Chicago, Illinois), and results were considered statistically significant when $P<.05$.

\section{Results}

During the study period, 389 patients attended the outpatient clinic, 170 of whom were undergoing long-term NIV treatment. Of these 170 patients, 5 were disabled (arthropathy), 3 had arrhythmias (heart rate $>120$ beats/ min), 9 were too obese to walk, 8 had severe dyspnea at the beginning of the 6MWT, and 1 refused to walk, and all these patients were excluded from the study. Ultimately, 144 subjects (91 male) were included in the study. Subjects were grouped according to their diagnoses, as follows: COPD $26.4 \%$ ( $n=38,6$ female), OHS $36.1 \%$ ( $n=52$, 28 female), kyphoscoliosis $16 \%$ ( $n=23,7$ female), and parenchymal lung diseases $21.5 \%$ ( $n=31,12$ female). Subject demographics and characteristics, spirometry, and ABG values according to underlying diseases and sex are shown in Table 1. The median values (IQR) of 6MWD, and percent-of-predicted 6MWD for all patients according to their underlying diseases were: COPD $316 \mathrm{~m} \mathrm{(226-390}$ $\mathrm{m})$, and 59.4\% (42.5-68.9\%); OHS $303 \mathrm{~m}$ (240-362 m), and $73.0 \%$ (63.0-82.0\%); kyphoscoliosis $420 \mathrm{~m} \mathrm{(318-}$ $462 \mathrm{~m}$ ), and $70.5 \%$ (56.0-75.2\%); parenchymal lung disease $333 \mathrm{~m}(273-372 \mathrm{~m})$, and $67.1 \%$ (46.7-74.7\%), respectively. Table 2 summarizes the subject groups according to underlying chronic respiratory diseases and sex relative to their 6MWT results and Borg dyspnea ${ }^{15}$ and fatigue scores. ${ }^{16}$

The correlation of actual 6MWD and percent-of-predicted 6MWD between lung function tests (spirometry) and $A B G$ values relative to the underlying chronic respiratory failure diseases (COPD, OHS, kyphoscoliosis, parenchymal lung disease) are shown in Table 3 . The sexspecific correlation of actual 6MWD and percent-of-predicted 6MWD between lung function tests (spirometry test) and ABG values are shown in Table 4. In males the correlation coefficient of percent-of-predicted 6MWD was greater than actual 6MWD in nearly all pre- and post-test parameters of 6MWT, as well as ABG and spirometry results (see Table 4). In females the correlation coefficient of actual 6MWD was greater (and the correlations were better) than percent-of-predicted 6MWD in pre- and posttest parameters of 6MWT (see Table 4). However, percent-of-predicted 6MWD values were significantly better 
The 6-Minute Walk Test in Chronic Respiratory Failure

Table 1. Subject Demographics, Spirometry, and Arterial Blood Gases Values by Disease and Sex

\begin{tabular}{|c|c|c|c|c|c|c|c|c|}
\hline & \multicolumn{2}{|c|}{$\begin{array}{l}\text { COPD } \\
n=38\end{array}$} & \multicolumn{2}{|c|}{$\begin{array}{l}\text { Obesity Hypoventilation } \\
\text { Syndrome } \\
n=52\end{array}$} & \multicolumn{2}{|c|}{$\begin{array}{c}\text { Kyphoscoliosis } \\
n=23\end{array}$} & \multicolumn{2}{|c|}{$\begin{array}{c}\text { Parenchymal Lung } \\
\text { Diseases } \\
n=31\end{array}$} \\
\hline & $\begin{array}{l}\text { Female } \\
n=6\end{array}$ & $\begin{array}{c}\text { Male } \\
n=32\end{array}$ & $\begin{array}{l}\text { Female } \\
n=28\end{array}$ & $\begin{array}{c}\text { Male } \\
n=24\end{array}$ & $\begin{array}{c}\text { Female } \\
n=7\end{array}$ & $\begin{array}{c}\text { Male } \\
n=16\end{array}$ & $\begin{array}{l}\text { Female } \\
n=12\end{array}$ & $\begin{array}{c}\text { Male } \\
n=19\end{array}$ \\
\hline Age, y & $67(60-70)$ & $64(56-72)$ & $69(59-75)$ & $61(57-78)$ & $46(38-55)$ & $49(44-55)$ & $72(64-76)$ & $63(49-72)$ \\
\hline BMI, $\mathrm{kg} / \mathrm{m}^{2}$ & $26(22-36)$ & $24(21-30)$ & $41(37-47)$ & $38(35-41)$ & $28(20-40)$ & $29(26-32)$ & $28(18-32)$ & $29(24-31)$ \\
\hline $\mathrm{pH}$ & $\begin{array}{c}7.46 \\
(7.45-7.47)\end{array}$ & $\begin{array}{c}7.45 \\
(7.41-7.49)\end{array}$ & $\begin{array}{c}7.44 \\
(7.41-7.46)\end{array}$ & $\begin{array}{c}7.45 \\
(7.41-7.48)\end{array}$ & $\begin{array}{c}7.45 \\
(7.42-7.48)\end{array}$ & $\begin{array}{c}7.44 \\
(7.41-7.47)\end{array}$ & $\begin{array}{c}7.45 \\
(7.39-7.51)\end{array}$ & $\begin{array}{c}7.43 \\
(7.39-7.48)\end{array}$ \\
\hline $\mathrm{P}_{\mathrm{aCO}_{2}}, \mathrm{~mm} \mathrm{Hg}$ & $46(44-46)$ & $49(44-54)$ & $46(42-50)$ & $48(40-52)$ & $49(45-60)$ & $50(42-53)$ & $45(41-49)$ & $49(44-57)$ \\
\hline $\mathrm{HCO}_{3}^{-}, \mathrm{mmol}$ & $\begin{array}{c}31.8 \\
(27.9-31.9)\end{array}$ & $\begin{array}{c}34.1 \\
(30.1-36.9)\end{array}$ & $\begin{array}{c}30.8 \\
(28.9-34.0)\end{array}$ & $\begin{array}{c}32.5 \\
(27.3-35.6)\end{array}$ & $\begin{array}{c}33.0 \\
(30.3-37.2)\end{array}$ & $\begin{array}{c}32.1 \\
(28.8-36.1)\end{array}$ & $\begin{array}{c}31.9 \\
(27.7-34.2)\end{array}$ & $\begin{array}{c}34.3 \\
(31.2-35.4)\end{array}$ \\
\hline $\mathrm{S}_{\mathrm{aO}_{2}}, \%$ & $92(90-95)$ & $93(91-95)$ & $93(92-94)$ & 93 (91-94) & $93(92-96)$ & $94(90-96)$ & $96(91-97)$ & $93(89-94)$ \\
\hline $\mathrm{P}_{\mathrm{aO}_{2}} / \mathrm{F}_{\mathrm{IO}_{2}}, \mathrm{~mm} \mathrm{Hg}$ & $\begin{array}{c}263 \\
(230-310)\end{array}$ & $\begin{array}{c}288 \\
(195-310)\end{array}$ & $\begin{array}{c}300 \\
(229-320)\end{array}$ & $\begin{array}{c}293 \\
(223-320)\end{array}$ & $\begin{array}{c}310 \\
(300-380)\end{array}$ & $\begin{array}{c}335 \\
(275-365)\end{array}$ & $\begin{array}{c}261 \\
(212-353)\end{array}$ & $\begin{array}{c}295 \\
(250-315)\end{array}$ \\
\hline $\mathrm{FEV}_{1}, \mathrm{~mL}^{*}$ & $\begin{array}{c}510 \\
(470-670)\end{array}$ & $\begin{array}{c}620 \\
(500-820)\end{array}$ & $\begin{array}{c}850 \\
(700-1150)\end{array}$ & $\begin{array}{c}1180 \\
(720-1770)\end{array}$ & $\begin{array}{c}445 \\
(340-605)\end{array}$ & $\begin{array}{c}790 \\
(590-920)\end{array}$ & $\begin{array}{c}680 \\
(515-740)\end{array}$ & $\begin{array}{c}810 \\
(580-1080)\end{array}$ \\
\hline$\%$ Predicted $\mathrm{FEV}_{1}, \% *$ & $35(32-36)$ & $24(17-30)$ & $51(38-63)$ & $44(28-60)$ & $33(25-39)$ & $30(18-35)$ & $41(27-45)$ & $28(21-40)$ \\
\hline $\mathrm{FVC}, \mathrm{mL}^{*}$ & $\begin{array}{c}970 \\
(910-1510)\end{array}$ & $\begin{array}{c}1340 \\
(820-1820)\end{array}$ & $\begin{array}{c}1060 \\
(870-1390)\end{array}$ & $\begin{array}{c}1620 \\
(1220-2040)\end{array}$ & $\begin{array}{c}480 \\
(350-690)\end{array}$ & $\begin{array}{c}880 \\
(810-1040)\end{array}$ & $\begin{array}{c}995 \\
(605-1025)\end{array}$ & $\begin{array}{c}1000 \\
(760-1400)\end{array}$ \\
\hline$\%$ Predicted FVC, $\% *$ & $56(55-67)$ & $37(25-51)$ & $49(36-67)$ & $47(34-61)$ & $30(21-37)$ & $26(22-33)$ & $47(27-50)$ & $30(21-38)$ \\
\hline $\mathrm{FEV}_{1} / \mathrm{FVC}^{*}$ & $\begin{array}{c}0.44 \\
(0.42-0.51)\end{array}$ & $\begin{array}{c}0.54 \\
(0.46-0.63)\end{array}$ & $\begin{array}{c}0.83 \\
(0.81-0.93)\end{array}$ & $\begin{array}{c}0.74 \\
(0.65-0.87)\end{array}$ & $\begin{array}{c}0.93 \\
(0.88-0.98)\end{array}$ & $\begin{array}{c}0.88 \\
(0.75-0.91)\end{array}$ & $\begin{array}{c}0.77 \\
(0.72-1.00)\end{array}$ & $\begin{array}{c}0.79 \\
(0.73-0.85)\end{array}$ \\
\hline
\end{tabular}

$\overline{\text { Values are median (IQR). }}$

* Values were obtained (female/male) in COPD 5/29, obesity hypoventilation syndrome 23/18, kyphoscoliosis 4/11, parenchymal lung diseases $12 / 19$, respectively.

BMI $=$ body mass index

$\mathrm{S}_{\mathrm{aO}_{2}}=$ arterial oxygen saturation

Table 2. 6-Minute Walk Test Data by Disease and Sex

\begin{tabular}{|c|c|c|c|c|c|c|c|c|}
\hline & \multicolumn{2}{|c|}{$\begin{array}{l}\text { COPD } \\
n=38\end{array}$} & \multicolumn{2}{|c|}{$\begin{array}{l}\text { Obesity Hypoventilation } \\
\text { Syndrome } \\
n=52\end{array}$} & \multicolumn{2}{|c|}{$\begin{array}{l}\text { Kyphoscoliosis } \\
\quad n=23\end{array}$} & \multicolumn{2}{|c|}{$\begin{array}{c}\text { Parenchymal Lung } \\
\text { Diseases } \\
n=31\end{array}$} \\
\hline & $\begin{array}{c}\text { Female } \\
n=6\end{array}$ & $\begin{array}{c}\text { Male } \\
n=32\end{array}$ & $\begin{array}{l}\text { Female } \\
n=28\end{array}$ & $\begin{array}{c}\text { Male } \\
n=24\end{array}$ & $\begin{array}{c}\text { Female } \\
n=7\end{array}$ & $\begin{array}{c}\text { Male } \\
n=16\end{array}$ & $\begin{array}{l}\text { Female } \\
n=12\end{array}$ & $\begin{array}{c}\text { Male } \\
n=19\end{array}$ \\
\hline Pre-test heart rate, beats/min & $94(87-102)$ & $97(89-108)$ & $88(82-97)$ & $88(82-97)$ & $87(84-90)$ & $95(87-105)$ & $96(89-106)$ & $86(74-110)$ \\
\hline Pre-test dyspnea score & $2(1-2)$ & $2(1-3)$ & $2(1-3)$ & $1(0-2)$ & $0(0-1)$ & $1(0-3)$ & $1(1-2)$ & $2(0-2)$ \\
\hline Pre-test fatigue score & $2(0-3)$ & $1(0-3)$ & $2(1-3)$ & $0(0-2)$ & $0(0-2)$ & $0(0-2)$ & $1(0-1)$ & $0(0-2)$ \\
\hline Pre-test $\mathrm{S}_{\mathrm{aO}_{2}}, \%$ & 97 (96-97) & 96 (94-97) & 95 (94-97) & $95(94-96)$ & $94(94-95)$ & $96(95-97)$ & $96(95-97)$ & $95(93-96)$ \\
\hline Post-test heart rate, beats/min & $\begin{array}{c}118 \\
(107-120)\end{array}$ & $\begin{array}{c}114 \\
(103-124)\end{array}$ & $\begin{array}{c}113 \\
(102-123)\end{array}$ & $\begin{array}{c}109 \\
(96-116)\end{array}$ & $\begin{array}{c}108 \\
(101-114)\end{array}$ & $\begin{array}{c}116 \\
(105-126)\end{array}$ & $\begin{array}{c}116 \\
(104-124)\end{array}$ & $\begin{array}{c}103 \\
(94-115)\end{array}$ \\
\hline Post-test dyspnea score & $3(3-4)$ & $3(1-5)$ & $3(1-5)$ & $2(0-3)$ & $1(1-3)$ & $1(1-4)$ & $2(2-3)$ & $2(1-4)$ \\
\hline Post-test fatigue score & $3(1-4)$ & $3(1-5)$ & $3(2-5)$ & $1(0-3)$ & $1(0-3)$ & $2(1-3)$ & $1(1-2)$ & $1(0-3)$ \\
\hline Post-test $\mathrm{S}_{\mathrm{aO}_{2}}, \%$ & 90 (79-93) & $90(86-94)$ & $91(87-93)$ & $92(89-93)$ & $88(80-90)$ & $87(84-92)$ & $92(89-93)$ & $89(86-93)$ \\
\hline Actual 6MWD & $\begin{array}{c}303 \\
(282-330)\end{array}$ & $\begin{array}{c}320 \\
(226-393)\end{array}$ & $\begin{array}{c}278 \\
(211-312)\end{array}$ & $\begin{array}{c}353 \\
(314-420)\end{array}$ & $\begin{array}{c}318 \\
(261-379)\end{array}$ & $\begin{array}{c}429 \\
(408-473)\end{array}$ & $\begin{array}{c}285 \\
(197-338)\end{array}$ & $\begin{array}{c}336 \\
(300-431)\end{array}$ \\
\hline Ideal 6MWD, m & $\begin{array}{c}459 \\
(418-501)\end{array}$ & $\begin{array}{c}537 \\
(507-595)\end{array}$ & $\begin{array}{c}357 \\
(336-388)\end{array}$ & $\begin{array}{c}491 \\
(450-527)\end{array}$ & $\begin{array}{c}522 \\
(456-671)\end{array}$ & $\begin{array}{c}635 \\
(585-692)\end{array}$ & $\begin{array}{c}445 \\
(397-488)\end{array}$ & $\begin{array}{c}532 \\
(478-624)\end{array}$ \\
\hline$\%$ Predicted 6MWD & $\begin{array}{c}60.9 \\
(56.3-71.9)\end{array}$ & $\begin{array}{c}59.4 \\
(41.6-67.5)\end{array}$ & $\begin{array}{c}74.6 \\
(63.2-84.3)\end{array}$ & $\begin{array}{c}68.7 \\
(61.9-81.7)\end{array}$ & $\begin{array}{c}61.9 \\
(45.1-75.9)\end{array}$ & $\begin{array}{c}72.2 \\
(61.5-74.6)\end{array}$ & $\begin{array}{c}61.7 \\
(46.5-74.0)\end{array}$ & $\begin{array}{c}67.7 \\
(59.1-74.8)\end{array}$ \\
\hline Lower limit of normal, $\mathrm{m}$ & $\begin{array}{c}320 \\
(279-362)\end{array}$ & $\begin{array}{c}384 \\
(354-442)\end{array}$ & $\begin{array}{c}218 \\
(197-249)\end{array}$ & $\begin{array}{c}338 \\
(297-374)\end{array}$ & $\begin{array}{c}383 \\
(317-532)\end{array}$ & $\begin{array}{c}482 \\
(432-539)\end{array}$ & $\begin{array}{c}306 \\
(258-349)\end{array}$ & $\begin{array}{c}379 \\
(325-471)\end{array}$ \\
\hline $\begin{array}{l}\text { Values are median (IQR). } \\
\mathrm{S}_{\mathrm{aO} 2}=\text { arterial oxygen saturation } \\
6 \mathrm{MWD}=6 \text {-minute walk distance }\end{array}$ & & & & & & & & \\
\hline
\end{tabular}


The 6-Minute Walk Test in Chronic Respiratory Failure

Table 3. Correlation of Actual and Predicted 6MWD With Other Variables

\begin{tabular}{|c|c|c|c|c|c|c|c|c|c|c|c|c|c|c|c|c|}
\hline & \multicolumn{4}{|c|}{$\begin{array}{l}\text { COPD } \\
n=38\end{array}$} & \multicolumn{4}{|c|}{$\begin{array}{c}\text { Obesity Hypoventilation } \\
\text { Syndrome } \\
n=52\end{array}$} & \multicolumn{4}{|c|}{$\begin{array}{l}\text { Kyphoscoliosis } \\
\quad n=23\end{array}$} & \multicolumn{4}{|c|}{$\begin{array}{l}\text { Parenchymal Lung Disease } \\
\qquad n=31\end{array}$} \\
\hline & \multicolumn{2}{|c|}{$\begin{array}{l}\text { Actual } \\
\text { 6MWD }\end{array}$} & \multicolumn{2}{|c|}{$\begin{array}{l}\text { Predicted } \\
6 \mathrm{MWD}\end{array}$} & \multicolumn{2}{|c|}{$\begin{array}{l}\text { Actual } \\
6 \mathrm{MWD}\end{array}$} & \multicolumn{2}{|c|}{$\begin{array}{l}\text { Predicted } \\
6 \mathrm{MWD}\end{array}$} & \multicolumn{2}{|c|}{$\begin{array}{l}\text { Actual } \\
6 \mathrm{MWD}\end{array}$} & \multicolumn{2}{|c|}{$\begin{array}{l}\text { Predicted } \\
6 \mathrm{MWD}\end{array}$} & \multicolumn{2}{|c|}{$\begin{array}{l}\text { Actual } \\
6 \mathrm{MWD}\end{array}$} & \multicolumn{2}{|c|}{$\begin{array}{l}\text { Predicted } \\
\text { 6MWD }\end{array}$} \\
\hline & $\mathrm{r}$ & $P$ & $\mathrm{r}$ & $P$ & $\mathrm{r}$ & $P$ & $\mathrm{r}$ & $P$ & $\mathrm{r}$ & $P$ & $\mathrm{r}$ & $P$ & $\mathrm{r}$ & $P$ & $\mathrm{r}$ & $P$ \\
\hline Pre-test heart rate & -0.44 & .006 & -0.44 & .006 & -0.9 & .50 & -0.28 & .047 & 0.20 & .37 & -0.03 & .89 & -0.56 & .001 & -0.64 & .001 \\
\hline Pre-test dyspnea & -0.37 & .02 & -0.46 & .004 & -0.42 & .002 & -0.23 & .11 & -0.33 & .12 & -0.33 & .12 & -0.03 & .86 & -0.18 & .32 \\
\hline Pre-test fatigue & -0.44 & .005 & -0.43 & .007 & -0.43 & .001 & -0.20 & .15 & -0.33 & .13 & -0.21 & .34 & -0.17 & .37 & -0.18 & .34 \\
\hline Pre-test $\mathrm{S}_{\mathrm{aO}_{2}}$ & 0.11 & .53 & 0.16 & .34 & 0.13 & .36 & 0.18 & .20 & 0.53 & .009 & 0.27 & .22 & -0.03 & .89 & 0.03 & .88 \\
\hline Post-test heart rate & -0.29 & .08 & -0.33 & .042 & -0.17 & .24 & -0.16 & .29 & 0.45 & .03 & 0.24 & .26 & -0.60 & .001 & -0.56 & .001 \\
\hline Post-test dyspnea & -0.56 & .001 & -0.61 & .001 & -0.54 & .001 & -0.34 & .01 & -0.32 & .14 & -0.25 & .25 & -0.27 & .14 & -0.34 & .06 \\
\hline Post-test fatigue & -0.50 & .001 & -0.50 & .002 & -0.60 & .001 & -0.39 & .004 & -0.22 & .33 & -0.08 & .73 & -0.21 & .25 & -0.17 & .36 \\
\hline Post-test $\mathrm{S}_{\mathrm{aO}_{2}}$ & 0.17 & .30 & 0.17 & .30 & 0.20 & .16 & 0.27 & .055 & 0.33 & .13 & 0.17 & .44 & 0.08 & .66 & 0.14 & .46 \\
\hline $\mathrm{P}_{\mathrm{aCO}_{2}}$ & -0.20 & .23 & -0.25 & .13 & -0.07 & .61 & -0.31 & .03 & -0.21 & .35 & -0.13 & .55 & -0.16 & .40 & -0.17 & .37 \\
\hline $\mathrm{P}_{\mathrm{aO}_{2}} / \mathrm{F}_{\mathrm{IO}_{2}}$ & 0.53 & .001 & 0.54 & .001 & 0.21 & .12 & 0.45 & .001 & 0.27 & .21 & 0.00 & .98 & 0.42 & .02 & 0.24 & .19 \\
\hline $\mathrm{FEV}_{1} *$ & 0.35 & .046 & 0.33 & .056 & 0.41 & .008 & 0.37 & .02 & 0.65 & .009 & 0.54 & .04 & 0.40 & .03 & 0.23 & .18 \\
\hline$\%$ Predicted $\mathrm{FEV}_{1} *$ & 0.26 & .13 & 0.40 & .02 & 0.17 & .28 & 0.47 & .002 & 0.23 & .41 & 0.46 & .08 & 0.14 & .46 & 0.26 & .16 \\
\hline $\mathrm{FVC}^{*}$ & 0.21 & .23 & 0.22 & .20 & 0.62 & .001 & 0.48 & .002 & 0.78 & .001 & 0.53 & .043 & 0.26 & .16 & 0.19 & .32 \\
\hline$\%$ Predicted FVC* & 0.07 & .71 & 0.22 & .22 & 0.30 & .055 & 0.55 & .001 & 0.29 & .30 & 0.55 & .03 & -0.02 & .94 & 0.12 & .54 \\
\hline $\mathrm{FEV}_{1} / \mathrm{FVC}^{*}$ & 0.37 & .03 & 0.30 & .08 & -0.26 & .10 & -0.04 & .80 & -0.32 & .25 & -0.18 & .53 & 0.06 & .75 & 0.06 & .76 \\
\hline $\begin{array}{l}6 \mathrm{MWD}=6 \text {-min walk dista } \\
\mathrm{r}=\text { correlation coefficient } \mathrm{o} \\
\mathrm{S}_{\mathrm{aO}_{2}}=\text { arterial oxygen satu }\end{array}$ & earn & . & & & & & & & & & & & & & & \\
\hline
\end{tabular}

Table 4. Correlation of Actual and Predicted 6MWD With Spirometry and Arterial Blood Gases

\begin{tabular}{|c|c|c|c|c|c|c|c|c|}
\hline & \multicolumn{4}{|c|}{ Actual 6MWD } & \multicolumn{4}{|c|}{ Predicted 6MWD } \\
\hline & \multicolumn{2}{|c|}{ Female } & \multicolumn{2}{|c|}{ Male } & \multicolumn{2}{|c|}{ Female } & \multicolumn{2}{|c|}{ Male } \\
\hline & $\mathrm{r}$ & $P$ & $\mathrm{r}$ & $P$ & $\mathrm{r}$ & $P$ & $\mathrm{r}$ & $P$ \\
\hline Pre-test heart rate & -0.22 & .12 & -0.30 & .003 & -0.26 & .07 & -0.47 & .001 \\
\hline Pre-test dyspnea score & -0.39 & .004 & -0.33 & .001 & -0.21 & .13 & -0.40 & .001 \\
\hline Pre-test fatigue score & -0.41 & .002 & -0.31 & .003 & -0.15 & .29 & -0.34 & .001 \\
\hline Pre-test $\mathrm{S}_{\mathrm{aO}_{2}}$ & 0.10 & .47 & 0.22 & .03 & 0.15 & .29 & 0.11 & .30 \\
\hline Post-test heart rate, beats/min & -0.24 & .08 & -0.14 & .19 & -0.17 & .22 & -0.31 & .003 \\
\hline Post-test dyspnea score & -0.51 & .001 & -0.42 & .001 & -0.28 & .04 & -0.50 & .001 \\
\hline Post-test fatigue score & -0.39 & .004 & -0.36 & .001 & -0.15 & .29 & -0.42 & .001 \\
\hline Post-test $\mathrm{S}_{\mathrm{aO}_{2}}$ & 0.04 & .79 & 0.13 & .22 & 0.25 & .07 & 0.18 & .08 \\
\hline $\mathrm{P}_{\mathrm{aCO}_{2}}$ & 0.02 & .86 & -0.27 & .01 & -0.14 & .31 & -0.30 & .004 \\
\hline $\mathrm{P}_{\mathrm{aO}_{2}} / \mathrm{F}_{\mathrm{IO}_{2}}$ & 0.29 & .03 & 0.53 & .001 & 0.24 & .09 & 0.46 & .001 \\
\hline $\mathrm{FEV}_{1}, \mathrm{~mL}^{*}$ & 0.18 & .23 & 0.32 & .005 & 0.54 & .001 & 0.42 & .001 \\
\hline$\%$ Predicted $\mathrm{FEV}_{1} *$ & 0.18 & .25 & 0.26 & .03 & 0.55 & .001 & 0.44 & .001 \\
\hline $\mathrm{FVC}, \mathrm{mL}^{*}$ & 0.20 & .20 & 0.12 & .30 & 0.40 & .007 & 0.25 & .03 \\
\hline$\%$ Predicted FVC* & 0.16 & .30 & 0.05 & .69 & 0.39 & .39 & 0.23 & .044 \\
\hline $\mathrm{FEV}_{1} / \mathrm{FVC}^{*}$ & -0.01 & .90 & 0.36 & .001 & 0 & .99 & 0.35 & .003 \\
\hline \multicolumn{9}{|c|}{$\begin{array}{l}\text { * Values were obtained in } 44 \text { females and } 77 \text { males. } \\
6 \mathrm{MWD}=6 \text {-min walk distance } \\
\mathrm{r}=\text { correlation coefficient of Spearman rank test }\end{array}$} \\
\hline
\end{tabular}

correlated with spirometry results than actual 6MWD in females (see Table 4).
The Figure shows the difference between the predicted and the observed 6MWD drawn against the predicted value 

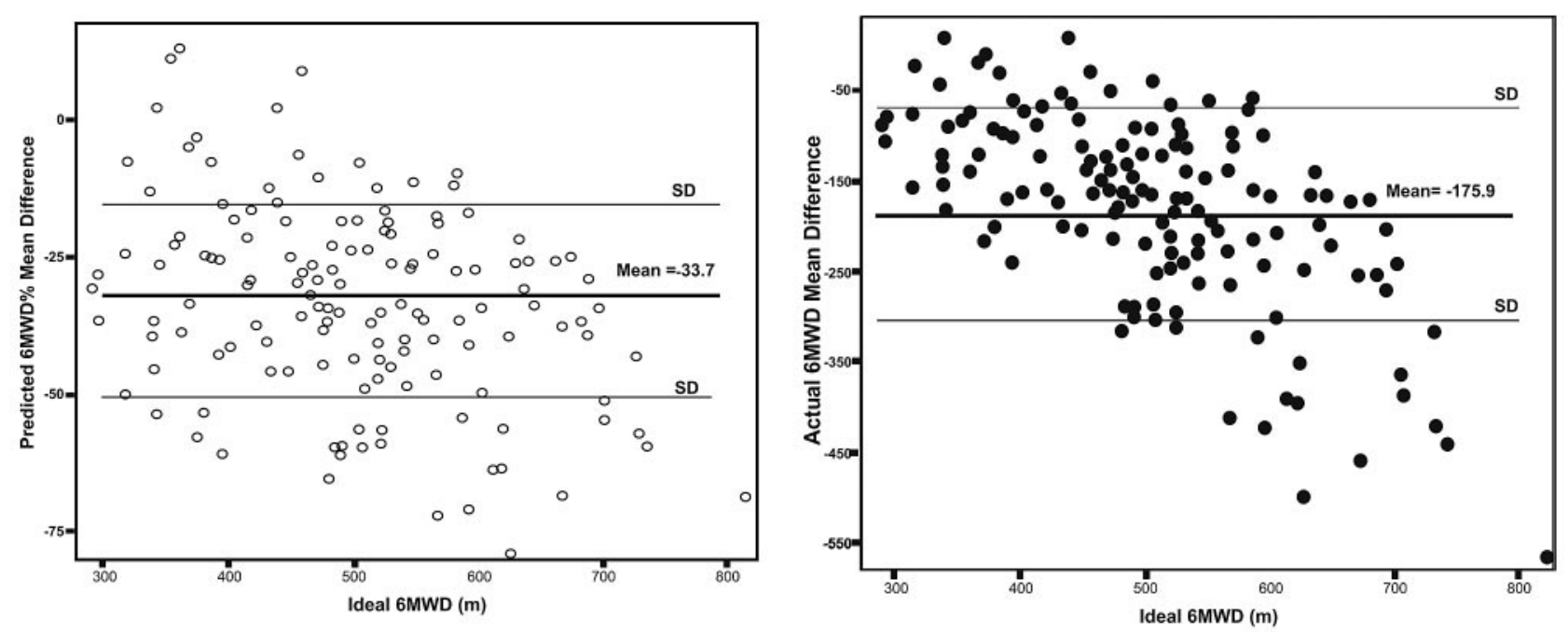

Figure. Scatter plot graphs of the difference between the percent-of-predicted value (on the left) and the actual 6-min walk distance (6MWD) (on the right) against the ideal value of 6MWD. Left: The mean \pm SD difference of the percent-of-predicted 6MWD was $-33.7 \pm 17.7$. Right: The mean \pm SD difference of the actual 6 MWD was $-175.9 \pm 110.4$.

Table 5. Standardized Residuals* Between Females and Males

\begin{tabular}{lcccccc}
\hline \hline & \multicolumn{3}{c}{$\begin{array}{c}\text { Residual of } \\
\text { Actual 6MWD } \\
(\mathrm{m})\end{array}$} & $P$ & $n$ & \multicolumn{2}{c}{$\begin{array}{l}\text { Residual of } \\
\% \text { Predicted } \\
\text { 6MWD }\end{array}$} & $P$ \\
\hline Female & 53 & $2.03 \pm 0.27$ & .002 & 53 & $0.12 \pm 1.05$ & .26 \\
Male & 91 & $1.97 \pm 0.25$ & & 91 & $-0.07 \pm 0.96$ &
\end{tabular}

* Differences between predicted and observed values were subtracted from the mean and divided to the standard deviation.

$6 \mathrm{MWD}=6$-minute walk distance

of 6MWD to investigate the residual distribution. The difference was uniformly distributed in percent-of-predicted 6MWD but varying in the actual 6MWD over the predicted ideal 6MWD. The Bland-Altman ${ }^{19}$ analysis was used to assess agreement between the 2 methods (actual 6MWD or percent-of-predicted 6MWD) and this is summarized in the Figure.

Residuals were compared between sexes to assess the potential bias in the congruity between the predicted and observed value of the 6MWD (ie, if the difference between the predicted and observed values were changed by these factors). These are summarized in Table 5. The residual was significant in actual 6MWD but not in percent-ofpredicted 6MWD $(P=.002$ and $P=.26$, respectively).

\section{Discussion}

In the present study, reference equations for 6MWD were used in a large series of subjects with chronic hypercapnic respiratory failure and undergoing home NIV treatment. In subjects using home NIV due to chronic hypercapnic respiratory failure because of COPD, OHS, or restrictive lung disease (kyphoscoliosis and parenchymal lung diseases), the percent-of-predicted 6MWD was more reflective of respiratory function than actual 6MWD.

Reference values for 6MWD have been studied in various healthy populations, and it was noted that demographic and anthropometric measurements are the most important variables in calculating reference equations. ${ }^{10,11,20-22} \mathrm{We}$ suggested that these equations may be better correlated with the functional parameters of patients with chronic respiratory failure. We used the correlation coefficient and Bland-Altman analysis ${ }^{19}$ to compare the actual 6MWD and the percent-of-predicted 6MWD and assess congruity between the 2 methods of clinical measurement. We found that percent-of-predicted 6MWD was a better method for assessing subjects' clinical status, with respect to sex. We found that the sex difference between predicted and actual values (residual difference) was not significant in percentof-predicted 6MWD, but significantly different in actual 6MWD.

According to Casanova and co-workers, age and sex are the most important variables when using 6MWD predictive equations in a large series of healthy subjects. The authors also showed geographical variations. ${ }^{11}$ In another study from North Africa, age, sex, weight, and height were similarly found to be factors affecting actual 6MWD, as was the number of parity among the females. ${ }^{10}$ In a further study, 6MWD for healthy Singaporean Chinese adults showed overestimated results when using equations derived from white subjects. ${ }^{20}$ Budweiser and co-workers ${ }^{7}$ studied the 6MWD in a population of subjects similar to those in our study. They used the same reference equations we did (the oldest and best known). ${ }^{8}$ They mainly assessed 
long-term survival and suggested a disease-specific use of 6MWD to assess and monitor NIV-treated subjects with chronic hypercapnic respiratory failure. ${ }^{7}$ They evaluated the 6MWD as a predictor of long-term survival for COPD $(n=197)$, restrictive lung disease $(n=112)$, and OHS $(n=115)$. Their results may be relevant to COPD, but have limited value for restrictive lung diseases. In the OHS group, 6MWD was found to be higher than in the other groups and not predictive of mortality. The authors calculated the percent-of-predicted 6MWD, but they did not use them for analysis. ${ }^{7}$ In the present study, although the majority of the subjects had mildly less hypercapnic and hypoxemic $A B G$ values and worse spirometry results, especially in OHS, they were more or less similar to the patients presented in the Budweiser report. Our study had higher 6MWD and predicted values. ${ }^{7}$

Age is an important determinant of 6MWD, and in many studies patients are included only when age is greater than 20 years or between $20-50$ or $40-85$ years. ${ }^{9,10,23-25}$ In the present study the majority of subjects were in their sixth decade, and the range was 32-85 years. Besides age, BMI is also crucial for reference equations in 6MWD. In our study the majority of the OHS group were morbidly obese $\left(\right.$ BMI $>40 \mathrm{~kg} / \mathrm{m}^{2}$ ), and the subjects with kyphoscoliosis and parenchymal diseases were overweight or nearly obese, but the COPD group had a normal BMI range. These characteristics were similar to those of the Budweiser study. ${ }^{7}$ Enright and Sherrill used all parameters for calculating reference values for $6 \mathrm{MWD}$ and cautioned using these with non-white patients and those younger than 40 or older than 80 years. ${ }^{8}$

Previous studies have shown that predicted $\mathrm{FEV}_{1}$ is highly correlated with 6MWD in patients with COPD, especially when $\mathrm{FEV}_{1}$ is $<35 \% .{ }^{26,27}$ However, these studies did not look at the relationship between percent-ofpredicted 6MWD values and spirometry results. In the present study the correlation of percent-of-predicted $\mathrm{FEV}_{1}$ was found to be significantly greater for percent-of-predicted 6MWD than actual 6MWD. We also showed that nearly all spirometry values in these OHS subjects using home NIV were significantly correlated with percent-ofpredicted 6MWD, compared with actual 6MWD. These findings indicate how important the percent-of-predicted 6MWD is for reflecting pulmonary function in obese subjects. Recently, Castro and co-workers evaluated the effect of hemodynamic status after 6 months of home NIV by 6MWD, spirometry, and echocardiography in OHS patients. ${ }^{28}$ They did not, however, assess the percent-of-predicted 6MWD in their statistical analysis.

There are some limitations in our study. The reference equations are designed for healthy subjects, 40-80 years of age and with a BMI $<35 \mathrm{~kg} / \mathrm{m}^{2}$. We applied these references to the subjects whose ages ranged between 32 85 years, and with a maximum BMI of $44 \mathrm{~kg} / \mathrm{m}^{2}$. We did not measure carbon monoxide lung diffusion capacity and lung volumes (total lung capacities or residual capacity). Also, although our data show predicted values in larger subjects with chronic respiratory failure undergoing home NIV treatment, our results cannot be applied to all disease groups.

\section{Conclusions}

In conclusion, by using reference equations, both actual and percent-of-predicted 6MWD are important for the assessment of cardiopulmonary functions. Sex is an important bias factor for the 6MWD when assessing functional status. The percent-of-predicted 6MWD appears to be better related to pulmonary function than the actual 6MWD alone.

\section{ACKNOWLEDGMENTS}

The authors would like to acknowledge and thank the American Thoracic Society Methods in Epidemiologic, Clinical, and Operations Research (MECOR) Program for assistance with this research and, in particular, Dr Ahmet Ugur Demir.

\section{REFERENCES}

1. ATS Committee on Proficiency Standards for Clinical Pulmonary Function Laboratories. ATS statement: guidelines for the six-minute walk test. Am J Respir Crit Care Med 2002;166(1):111-117.

2. Guyatt GH, Thompson PJ, Berman LB, Sullivan MJ, Townsend M, Jones NL, Pugsley SO. How should we measure function in patients with chronic heart and lung disease? J Chronic Dis 1985;38(6):517524.

3. Weisman IM, Zeballos RJ. An integrated approach to the interpretation of cardiopulmonary exercise testing. Clin Chest Med 1994; 15(2):421-445.

4. Martinez FJ, Foster G, Curtis JL, Criner G, Weinmann G, Fishman A, et al; NETT Research Group. Predictors of mortality in patients with emphysema and severe airflow obstruction. Am J Respir Crit Care Med 2006;173(12):1326-1334.

5. Miyamoto S, Nagaya N, Satoh T, Kyotani S, Sakamaki F, Fujita M, et al. Clinical correlates and prognostic significance of six-minute walk test in patients with primary pulmonary hypertension. Comparison with cardiopulmonary exercise testing. Am J Respir Crit Care Med 2000;161(2 Pt1):487-492.

6. Pinto-Plata VM, Cote C, Cabral H, Taylor J, Celli BR. The 6-min walk distance: change over time and value as a predictor of survival in severe COPD. Eur Respir J 2004;23(1):28-33.

7. Budweiser S, Heidtkamp F, Jörres RA, Heinemann F, Arzt M, Schroll $\mathrm{S}$, et al. Predictive significance of the six-minute walk distance for long-term survival in chronic hypercapnic respiratory failure. Respiration 2008;75(4):418-426.

8. Enright PL, Sherrill DL. Reference equations for the six-minute walk in healthy adults. Am J Respir Crit Care Med 1998;158(5 Pt 1): 1384-1387.

9. Troosters T, Gosselink R, Decramer M. Six minute walking distance in healthy elderly subjects. Eur Respir J 1999;14(2):270-274.

10. Ben Saad H, Prefaut C, Tabka Z, Mtir AH, Chemit M, Hassaoune R, et al. 6-minute walk distance in healthy North Africans older than 40 years: influence of parity. Respir Med 2009;103(1):74-84. 


\section{The 6-Minute Walk Test in Chronic Respiratory Failure}

11. Casanova C, Celli BR, Barria P, Casas A, Cote C, de Torres JP, et al. The 6-min walk distance in healthy subjects: reference standards from seven countries. Eur Respir J 2011;37(1):150-156.

12. Karakurt Z, Güven AO, Moçin OY, Karavelioğlu Y, Güngör G, Altınöz H, et al. Six minute walking distance in kyphoscoliosis patients with chronic respiratory value. Multidiscip Respir Med 2010; 5(4):244-249.

13. Global Initiative for Chronic Obstructive Lung Disease. Global strategy for the diagnosis, management, and prevention of chronic obstructive pulmonary disease. Update 2011. http://www.goldcopd.org. Accessed March 1, 2013.

14. Olson AL, Zwillich C. The obesity hypoventilation syndrome. Am J Med 2005;118(9):948-956.

15. Borg GA. Psychophysical bases of perceived exertion. Med Sci Sports Exerc 1982;14(5):377-381.

16. Wilson RC, Jones PW. A comparison of the visual analogue scale and modified Borg scale for the measurement of dyspnoea during exercise. Clin Sci (Lond) 1989;76(3):277-282.

17. Parker JM, Dillard TA, Phillips YY. Arm span-height relationships in patients referred for spirometry. Am J Respir Crit Care Med 1996;154(2 Pt 1):533-536.

18. Standardization of spirometry, 1994 update. American Thoracic Society. Am J Respir Crit Care Med 1995;152(3):1107-1136.

19. Bland JM, Altman DG. Statistical methods for assessing agreement between two methods of clinical measurement. Lancet 1986;1(8476): 307-310.

20. Poh H, Eastwood PR, Cecins NM, Ho KT, Jenkins SC. Six-minute walk distance in healthy Singaporean adults cannot be predicted using reference equations derived from Caucasian populations. Respirology 2006;11(2):211-216.

21. Alameri H, Al-Majed S, Al-Howaikan A. Six-min walk test in a healthy adult Arab population. Respir Med 2009;103(7):1041-1046.

22. Iwama AM, Andrade GN, Shima P, Tanni SE, Godoy I, Dourado VZ. The six-minute walk test and body weight-walk distance product in healthy Brazilian subjects. Braz J Med Biol Res 2009;42(11): 1080-1085.

23. Gibbons WJ, Fruchter N, Sloan S, Levy RD. Reference values for a multiple repetition 6-minute walk test in healthy adults older than 20 years. J Cardiopulm Rehabil 2001;21(2):87-93.

24. Camarri B, Eastwood PR, Cecins NM, Thompson PJ, Jenkins S. Six minute walk distance in healthy subjects aged 55-75 years. Respir Med 2006;100(4):658-665

25. Chetta A, Zanini A, Pisi G, Aiello M, Tzani P, Neri M, Oliveri D. Reference values for the 6-min walk test in healthy subjects 20-50 years old. Respir Med 2006;100(9):1573-1578.

26. Castro-Añón O, Golpe R, Pérez-de-Llano LA, López González MJ, Escalona Velasquez E, Pérez Fernández R et al. Haemodynamic effects of non-invasive ventilation in patients with obesity-hypoventilation syndrome. Respirology 2012;17(8):1269-1274. DOI: 10.1111/ j.1440-1843.2012.02252.x.

27. Inal-Ince D, Savci S, Coplu L, Arikan H. Functional capacity in severe chronic obstructive pulmonary disease. Saudi Med J 2005; 26(1):84-89.

28. Waatevik M, Johannessen A, Hardie JA, Bjordal JM, Aukrust P, Bakke PS, Eagan TM. Different COPD disease characteristics are related to different outcomes in the 6-minute walk test. COPD 2012; 9(3):227-234 